\title{
Reversal of the Weak Measurement of a Quantum State in a Superconducting Phase Qubit
}

\author{
Nadav Katz, ${ }^{1, *}$ Matthew Neeley, ${ }^{1}$ M. Ansmann, ${ }^{1}$ Radoslaw C. Bialczak, ${ }^{1}$ M. Hofheinz, ${ }^{1}$ Erik Lucero, ${ }^{1}$ A. O'Connell, ${ }^{1}$ \\ H. Wang, ${ }^{1}$ A. N. Cleland, ${ }^{1}$ John M. Martinis, ${ }^{1}$ and Alexander N. Korotkov ${ }^{2}$ \\ ${ }^{1}$ Department of Physics, University of California, Santa Barbara, California 93106, USA \\ ${ }^{2}$ Department of Electrical Engineering, University of California, Riverside, California 92521, USA
}

(Received 11 June 2008; published 10 November 2008)

\begin{abstract}
We demonstrate in a superconducting qubit the conditional recovery (uncollapsing) of a quantum state after a partial-collapse measurement. A weak measurement extracts information and results in a nonunitary transformation of the qubit state. However, by adding a rotation and a second partial measurement with the same strength, we erase the extracted information, canceling the effect of both measurements. The fidelity of the state recovery is measured using quantum process tomography and found to be above $70 \%$ for partial-collapse strength less than 0.6 .
\end{abstract}

The observation of a quantum system necessarily perturbs the state of the system. For a strong measurement, the quantum state is understood to collapse irrevocably to one of the eigenstates of the measurement operator; this concept of projective measurement is a central paradigm of modern physics [1]. For weak measurements, however, the collapse is now understood to be partial, with correspondingly partial information drawn from the measurement yielding a nonunitary, nonprojective transformation of the quantum state. It has been predicted (though never demonstrated previously) that after such a weak measurement, the initial quantum state of the system can be recovered by essentially undoing the effect of the measurement $[2,3]$ and causing a quantum "uncollapsing."

Superconducting phase qubits provide an excellent system for testing this concept of uncollapsing. Our experimental implementation [4] uses a controlled measurement process whose projective strength can be tuned continuously from a weak partial measurement to a full projective one [5]. Using this system, we can experimentally test reversing the partial, measurement-induced collapse of a quantum state. Similar tests of partial or continuous weak measurements should also be possible for other types of solid-state qubits [6].

In our experiment, the superconducting phase qubit is prepared in a combination of its ground $|0\rangle$ and first excited |1) states. A partial measurement of the qubit then yields a "detection" event, which occurs with probability $p$ when the qubit is in the $|1\rangle$ state, while it never occurs for the qubit in the $|0\rangle$ state. If the measurement yields a null result (i.e., no event detected), this leads to the partial collapse of the qubit state towards $|0\rangle$. This evolution towards the $|0\rangle$ state is driven by the extracted information, and does not involve any energy exchange. We then employ the following method (proposed by Jordan and one of the authors [3]) to "uncollapse" the result of the measurement [see pulse sequence in Fig. 1(d)]: After the preparation of an arbitrary initial state of the qubit and (i) partial collapse due to null- result measurement with strength $p$, we (ii) apply a $\pi$ pulse, coherently swapping the amplitudes of the qubit states $|0\rangle$ and $|1\rangle$, and (iii) partially measure the qubit state again, with the same measurement strength $p$ [7]. The combination of steps (ii) and (iii) "antisymmetrizes" the information extracted from the first measurement of the qubit, and with an overall probability $1-p$ of two null results [8], regardless of the initial state, the qubit state is coherently restored to its initial, premeasurement state (here including a $\pi$ rotation [7,9]).

In order for the uncollapsing procedure to work, we have to erase the information that was already extracted classi-
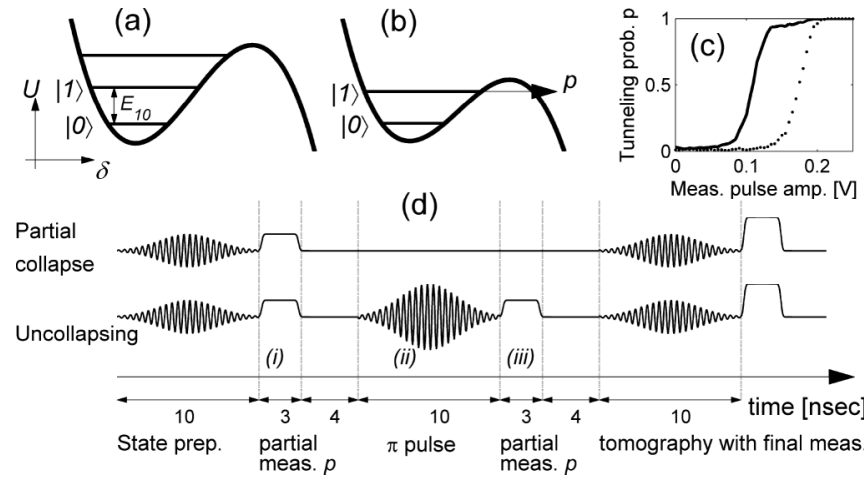

FIG. 1. (a) The qubit potential during application of the microwave pulses, which cause coherent transitions between states $|0\rangle$ and $|1\rangle$. (b) During a partial measurement the state $|1\rangle$ tunnels out of the well with probability $p$. (c) The tunneling probability $p$ (solid line) is determined by the amplitude of the measurement pulse which lowers the barrier; we use sufficiently small amplitude to avoid tunneling from the state $|0\rangle$ (dotted line). The maximal measurement visibility (the difference between the solid and dotted lines) is about 90\%. (d) Pulse sequences (including state tomography) for the partial-collapse experiment (upper trace, as in [5]) and for the quantum uncollapsing (lower trace). The effect of the partial measurement [step (i)] is undone by applying the $\pi$ pulse [step (ii)] and additional (uncollapsing) partial measurement [step (iii)] with the same strength $p$. 
cally. This distinguishes this measurement-induced uncollapsing from a "quantum eraser" [10], in which only potentially extractable information is erased. Note also that the result of the second measurement is stochastic, and only a particular result will succeed in erasing the information, thus leading to a less than unity probability of success for uncollapsing; however, in the case of this desired result, the qubit's initial state is fully recovered.

In this Letter, we report the first experimental demonstration of quantum uncollapsing by implementing the above described protocol, where we obtain fidelities well over $70 \%$, quantified by quantum process tomography [11]. Besides confirming the ability to undo a partial quantum measurement, this result confirms the high quantum efficiency of our measurement.

Our superconducting phase qubit has been described in detail previously [4]. We briefly review the relevant details and modifications here. The qubit is fabricated as a superconducting loop interrupted by a $\sim 1 \mu \mathrm{m}^{2}$-sized Josephson junction of critical current $2 \mu \mathrm{A}$, shunted by a low losstangent parallel plate capacitor $(1 \mathrm{pF})$ formed with a-Si:H dielectric. We initialize the system in the ground state of the qubit's cubic-shaped potential well [see Fig. 1(a)]. The logic qubit is formed by the ground state $|0\rangle$ and the first excited state $|1\rangle$ of this well (separated by $E_{10} / h=$ $6.75 \mathrm{GHz}$, with $h$ Planck's constant). A coherent initial state is prepared by a shaped (in both phase and amplitude) microwave pulse with nanosecond time resolution and 14 bit precision. We use on-resonance [12] $10 \mathrm{~ns}$ long, $4 \mathrm{~ns}$ FWHM, Slepian pulses $[13,14]$ to ensure optimal spectral properties (minimizing unwanted excitation of higher states of the well [14]) while avoiding pulse overlap in the time domain. The resulting (initial) qubit state can be written as

$$
\left|\psi_{0}\right\rangle=\cos \left(\theta_{0} / 2\right)|0\rangle+e^{-i \phi_{0}} \sin \left(\theta_{0} / 2\right)|1\rangle .
$$

The partial measurement [step (i) of the protocol] is done in the same way as in Ref. [5]. By applying a short ( $3 \mathrm{~ns}$ ) bias pulse, we lower the quantum well barrier [Fig. 1 (b)] that leads to the selective tunneling of the $|1\rangle$ state out of the well. The probability $p$ for this tunneling to occur (i.e., the measurement strength) can be tuned continuously from 0 to 1 by varying the bias pulse amplitude [Fig. 1(c)]. The tunneling event is registered at a later time with an onchip SQUID that easily distinguishes between states remaining in the qubit well and those that tunneled out. For the initial state given by Eq. (1), the tunneling occurs with probability $p \sin ^{2}\left(\theta_{0} / 2\right)$. If no tunneling occurs (null result), the initial state $\left|\psi_{0}\right\rangle$ changes (partially collapses) to

$$
\begin{gathered}
\left|\psi_{M}\right\rangle=\cos \left(\theta_{M} / 2\right)|0\rangle+e^{-i\left(\phi_{0}+\phi_{M}\right)} \sin \left(\theta_{M} / 2\right)|1\rangle, \\
\theta_{M}=2 \tan ^{-1}\left[\sqrt{1-p} \tan \left(\theta_{0} / 2\right)\right],
\end{gathered}
$$

where $\phi_{M}$ is an accumulated phase due to an adiabatic change in the energy level spacing during the measurement (in the language of generalized quantum measurements [11] the corresponding Kraus operator is $|0\rangle \times$ $\left.\left\langle 0\left|+e^{-i \phi_{M}} \sqrt{1-p}\right| 1\right\rangle\langle 1|\right)$. This information-related nonunitary transformation (confirmed in the experiment [5]) is precise only in the ideal case. It neglects energy and phase relaxation within the qubit well, which is an acceptable approximation since the corresponding relaxation times $T_{1}=450 \mathrm{~ns}$ and $T_{2}^{*}=350 \mathrm{~ns}$ (and $T_{2}=120 \mathrm{ns)} \mathrm{are} \mathrm{sig-}$ nificantly longer than the experiment duration [15]. Equations (2) and (3) also neglect incoherence and noise in the process of virtual tunneling; however, the theoretical analysis [16] confirms that the result (2) and (3) is a good approximation.

The qubit state after the partial collapse is analyzed by state tomography (as in [5,17-19]), consisting of 3 types of tomographic rotations (either a $\pi / 2$ pulse rotating about the $Y$ axis of the Bloch sphere, a $\pi / 2$ pulse rotating about the $X$ axis, or no rotation) followed by a full measurement (with $p \approx 1$ ) - see the upper trace in Fig. 1(d). In this way we measure the qubit tunneling probabilities $P_{X}, P_{Y}$, and $P_{Z}$, which correspond to the qubit state components $X, Y$, and $Z$ on the Bloch sphere (in the rotating frame). Since $P_{X}, P_{Y}$, and $P_{Z}$ include both the probability of tunneling during the tomography measurement and the background probability $P_{B}=p \sin ^{2}\left(\theta_{0} / 2\right)$ accumulated during the partial measurement [20], the qubit state components are given by $\{X,-Y,-Z\}=2\left(P_{\{X, Y, Z\}}-P_{B}\right) /\left(1-P_{B}\right)-1$ (the minus signs on $Y$ and $Z$ come from following the convention setting the $|0\rangle$ at $Z=+1$ ). The measured tunneling probabilities $P_{X}, P_{Y}$, and $P_{Z}$ for the initial state $(|0\rangle+|1\rangle) / \sqrt{2}$ are shown in Fig. 2(a), as functions of the pulse amplitude for partial measurement (which is in a oneto-one correspondence with $p$ ); in this case $P_{B}=p / 2$

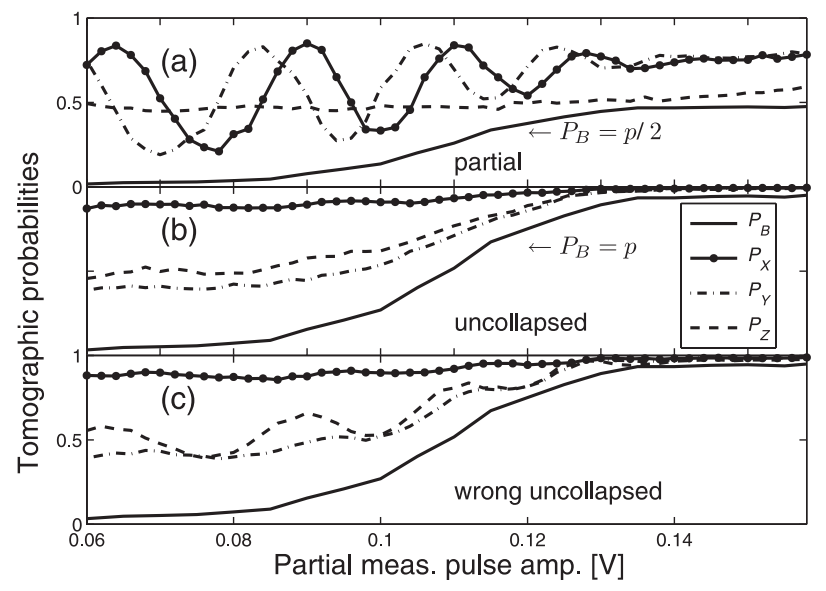

FIG. 2. The qubit tunneling probabilities $P_{X}, P_{Y}$, and $P_{Z}$ after the partial and tomographic $(X, Y, Z)$ measurements for (a) the partial-collapse sequence, (b) the uncollapsing sequence, and (c) a "wrong" uncollapsing with $\pi$ pulse replaced by $0.9 \pi$ pulse. Initial state is $(|0\rangle+|1\rangle) / \sqrt{2}$. The background $P_{B}$ is the probability of qubit tunneling before the state tomography (see text). 
[also shown in Fig. 2(a)]. Note the large oscillations in $P_{X}$ and $P_{Y}$, indicating that the partial measurement is accumulating a significant phase $\phi_{M}$, as was seen in [5]. The qubit state components $X, Y$, and $Z$ calculated from the data in Fig. 2(a) are shown on the Bloch sphere in Fig. 3(c).

In order to recover the initial quantum state, we now add steps (ii) and (iii) of the uncollapsing protocol-see the lower trace in Fig. 1(d). The $\pi$ pulse about the $X$ axis [step (ii)] after the partial collapse exchanges the basis states in Eq. (2), creating the qubit state $\left|\psi_{\pi}\right\rangle=\sin \left(\theta_{M} / 2\right)|0\rangle+$ $e^{i\left(\phi_{0}+\phi_{M}\right)} \cos \left(\theta_{M} / 2\right)|1\rangle$. The second partial measurement with the same strength $p$ [step (iii)] can either result in a tunneling event, or not. In the case of no tunneling (null result again) the partial-collapse evolution $\left|\psi_{\pi}\right\rangle \rightarrow\left|\psi_{F}\right\rangle$ is described by the same transformation as $\left|\psi_{0}\right\rangle \rightarrow\left|\psi_{M}\right\rangle$ [see Eqs. (1)-(3)]; it is easy to see that this produces the state $\left|\psi_{F}\right\rangle=\sin \left(\theta_{0} / 2\right)|0\rangle+e^{i \phi_{0}} \cos \left(\theta_{0} / 2\right)|1\rangle$. As expected, $\left|\psi_{F}\right\rangle$ coincides with the initial state $\left|\psi_{0}\right\rangle$ up to a $\pi$ rotation about the $X$ axis. Notice that not only the polar angle $\theta_{0}$ is restored (which is essentially the uncollapsing), but the azimuth angle shift $\phi_{M}$ is also canceled (due to the usual spin echo effect).

The state tomography of the uncollapsed state $\left|\psi_{F}\right\rangle$ is done in the same way as for the partially collapsed state $\left|\psi_{M}\right\rangle$. The only difference is that now the background probability $P_{B}$ is due to both partial measurements, and therefore $\quad P_{B}=1-\left[1-p \sin ^{2}\left(\theta_{0} / 2\right)\right][1-$ $\left.p \cos ^{2}\left(\theta_{M} / 2\right)\right]=p$, independent of the initial state. The measured probabilities $P_{X}, P_{Y}$, and $P_{Z}$ for the initial state $(|0\rangle+|1\rangle) / \sqrt{2}$ are shown in Fig. 2(b) as functions of the

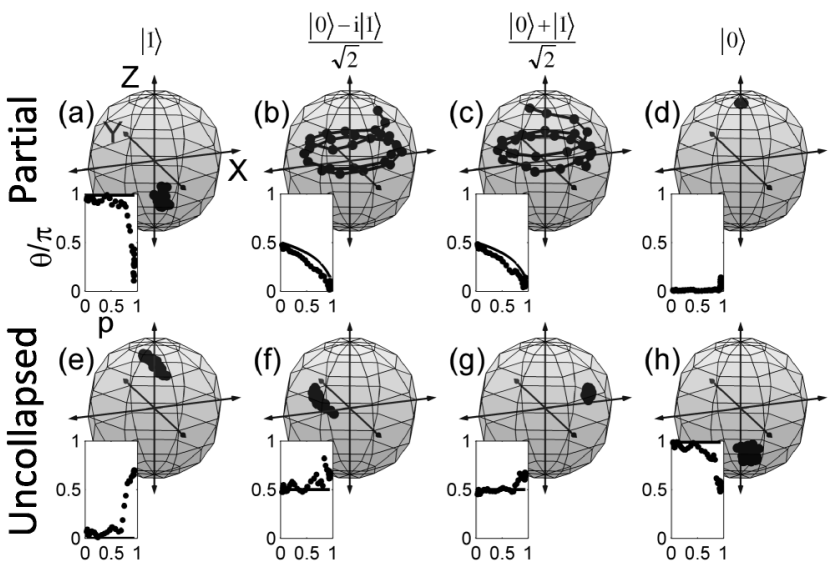

FIG. 3. The qubit states on the Bloch sphere, as measured by the state tomography, after the partial collapse (first row) and uncollapsing (second row). Initial state is $|1\rangle$ for panels (a) and (e), $(|0\rangle-i|1\rangle) / \sqrt{2}$ for (b) and (f), $(|0\rangle+|1\rangle) / \sqrt{2}$ for (c) and $(\mathrm{g})$, and $|0\rangle$ for (d) and (h). The points shown on the Bloch spheres (connected by lines as a guide for the eye) correspond to varied measurement strength up to $p=0.7$. For ideal uncollapsing the states in the second row should not depend on $p$. The insets in each panel show the measured (dots) and theoretical (line) dependence of the polar angle $\theta / \pi$ on $p$. measurement pulse amplitude, and the corresponding qubit states on the Bloch sphere are shown in Fig. 3(g). Notice that compared to the partial-collapse results, the oscillations in $P_{X}$ and $P_{Y}$ are clearly suppressed (spin echo) and the qubit state is restored to the equatorial plane. The measured state is quite close to the ideal result of uncollapsing $(|0\rangle+|1\rangle) / \sqrt{2}$ for $p \leq 0.6$ (see below). If we purposefully change the $\pi$ pulse of the step (ii) to a $0.9 \pi$ pulse, the oscillations of $P_{Y}$ and $P_{Z}$ are somewhat recovered [see Fig. 2(c)], and the qubit state moves significantly out of the equatorial plane (not shown), indicating that the uncollapsing procedure performance is degraded.

So far we have discussed experimental uncollapsing of the initial state $(|0\rangle+|1\rangle) / \sqrt{2}$. However, any initial state should be restored by the same procedure. Instead of examining all initial states to check this fact, it is sufficient to choose 4 initial states with linearly independent density matrices and use the linearity of quantum operations [11]. We choose initial states $|1\rangle,(|0\rangle-i|1\rangle) / \sqrt{2}, \quad(|0\rangle+$ $|1\rangle) / \sqrt{2}$, and $|0\rangle$. The corresponding qubit states on the Bloch sphere after the partial collapse and after uncollapsing are shown in Fig. 3 for measurements with a range of measurement strength $p$. For clarity we only show the results for $p \leq 0.7$, since beyond this range our simple theory becomes too inaccurate. The main reason why the protocol begins to fail for large $p$ is a noticeable probability $p_{r} \sim 0.1$ of energy relaxation to the ground state during our $44 \mathrm{~ns}$ long sequence $\left(T_{1}=450 \mathrm{~ns}\right)$. The relative contribution of such cases increases with $p$ and becomes very significant when the selection probability $1-p$ becomes comparable to $p_{r}$, thus ruining the fidelity of uncollapsing. Also notice that we use the experimentally determined $p$, as shown in 1(c), which contains an approximate 5\% error due to state preparation and measurement infidelities. The data are not rescaled to correct for this error.

Uncollapsing of the states $|0\rangle$ and $|1\rangle$ is straightforward (they do not change in null-result measurements), so the deviations from the ideal results in the left and right columns of Fig. 3 characterize the imperfections of our experiment. Uncollapsing of the states $(|0\rangle-i|1\rangle) / \sqrt{2}$ and $(|0\rangle+|1\rangle) / \sqrt{2}$ is non-trivial; however, we see that the small deviations in Figs. 3(f) and 3(g) from the theoretical result are approximately the same as for the trivial cases, thus indicating that the uncollapsing itself is nearly ideal. Besides the Bloch spheres, in Figs. 3(a)-3(h) we also show the dependence of the corresponding polar angles $\theta$ on the measurement strength $p$. The small discrepancy with the theory (with no fit parameters) is mainly due to intrinsic decoherence of the qubit and measurement error. As discussed above, the discrepancy becomes significant when $p$ approaches 1 .

The state tomography for these four initial states is sufficient for full characterization of the quantum process tomography (QPT) [11,21-23]. In Fig. 4(a) we show the QPT matrix $\chi$ in the standard Pauli-matrix basis $\left(I, \sigma_{X}, \sigma_{Y}, \sigma_{Z}\right)$, 

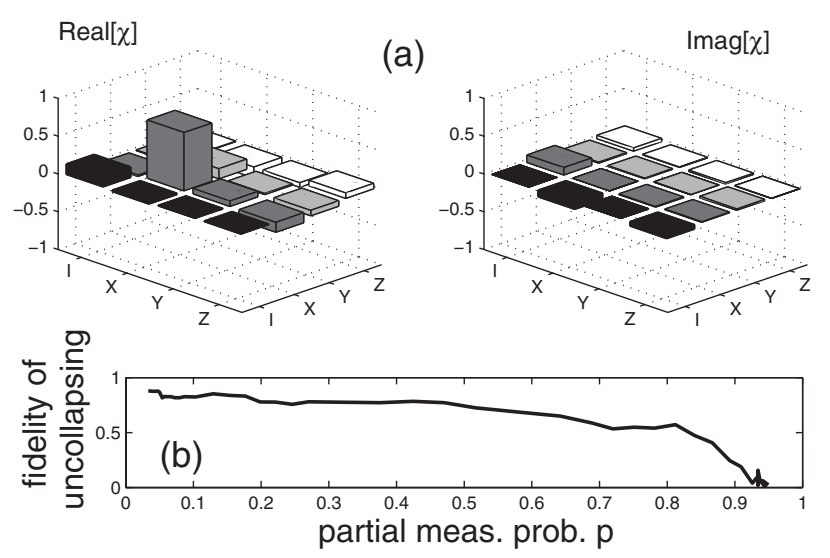

FIG. 4. (a) The quantum process tomography matrix $\chi$ for the uncollapsing with $p=0.47$. (b) The fidelity of the quantum uncollapsing as a function of the partial measurement probability $p$ [25].

generated by applying the conventional linear algebra formalism [11] to our results [24] for the uncollapsing protocol with $p=0.47$. As expected, we see a clear peak at the $(X, X)$ location, indicating that the process is mainly that of a $\pi$ rotation about the $X$ axis. The uncollapsing fidelity is defined as the overlap of the $\chi$ matrix with the ideal one (of a perfect $\pi$ pulse); i.e., the fidelity is simply $\operatorname{Re} \chi(X, X)$. The dependence of this fidelity on the measurement strength $p$ is presented in Fig. 4(b), which shows that the uncollapsing fidelity remains above $70 \%$ until the degradation of the state recovery at $p \gtrsim 0.6$.

In conclusion, we demonstrate a conditional uncollapsing of a partially measured quantum state, and quantify this process by quantum process tomography. While our protocol has apparent similarity with the spin echo sequence (and includes the azimuth angle recovery due to the echo effect), we emphasize the clear difference between the two effects: the spin echo is the undoing of an unknown unitary transformation, while uncollapsing is the undoing of a known but nonunitary transformation.

The authors thank A. Jordan for useful discussions. Devices were made at the UCSB Nanofabrication Facilities, a part of the NSF funded NNIN network. This work was supported by NSA/IARPA/ARO Grant No. W911NF-04-1-0204 and by NSF Grant No. CCF0507227.

*Current address: The Racah Institute of Physics, The Hebrew University, Jerusalem, 91904, Israel.

[1] Quantum Theory and Measurement, edited by J.A. Wheeler and W.H. Zurek (Princeton University Press, Princeton, NJ, 1983).

[2] M. Koashi and M. Ueda, Phys. Rev. Lett. 82, 2598 (1999); M. A. Nielsen and C. M. Caves, Phys. Rev. A 55, 2547
(1997); H. Mabuchi and P. Zoller, Phys. Rev. Lett. 76, 3108 (1996).

[3] A. N. Korotkov and A. N. Jordan, Phys. Rev. Lett. 97, 166805 (2006); A. N. Jordan and A. N. Korotkov, in Coherence and Quantum Optics IX, edited by N.P. Bigelow et al. (Optical Soc. of America, 2008), p. 191.

[4] J. M. Martinis et al., Phys. Rev. Lett. 89, 117901 (2002); K. B. Cooper et al., Phys. Rev. Lett. 93, 180401 (2004).

[5] N. Katz et al., Science 312, 1498 (2006).

[6] T. Yamamoto et al., Nature (London) 425, 941 (2003); J. H. Plantenberg et al., Nature (London) 447, 836 (2007); J. Majer et al., Nature (London) 449, 443 (2007); J. R. Petta et al., Science 309, 2180 (2005).

[7] The original proposal [3] called for another $\pi$ pulse to return to the original basis. Without loss of generality, we forgo this pulse in order to shorten the overall sequence.

[8] This probability coincides with the upper bound for the uncollapsing success probability [3]. It obviously decreases with increasing strength $p$ of the partial measurement, reaching zero for the projective collapse $(p=1)$.

[9] Erasing the information is only a necessary condition for uncollapsing; we also have to use a measurement with $100 \%$ quantum efficiency (so that quantum information does not leak to an unmeasurable environment) and compensate for a possible unitary transformation.

[10] M. O. Scully and K. Drühl, Phys. Rev. A 25, 2208 (1982).

[11] M. A. Nielsen and I. L. Chuang, Quantum Computation and Quantum Information (Cambridge University Press, Cambridge, England, 2000).

[12] Short pulses lead to power dependent phase shifts [as measured by Frederick W. Strauch, et al., IEEE Trans. Appl. Supercond. 17, 105 (2007)]. These shifts were compensated for by a $+8 \mathrm{MHz}$ detuning of the $\pi$ pulses, while no shifts were needed for the $\pi / 2$ pulses.

[13] D. Slepian, Bell Syst. Tech. J. 57, 1371 (1978).

[14] Erik Lucero et al., Phys. Rev. Lett. 100, 247001 (2008).

[15] Because of the similarity of the uncollapsing to the echo sequence, it is $T_{2}^{*}$ that determines the time scale for decay due to dephasing.

[16] L. P. Pryadko and A. N. Korotkov, Phys. Rev. B 76, 100503(R) (2007).

[17] M. Steffen et al., Phys. Rev. Lett. 97, 050502 (2006).

[18] A. A. Houck et al., Nature (London) 449, 328 (2007).

[19] Yu-xi Liu, L. F. Wei, and F. Nori, Europhys. Lett. 67, 874 (2004); Phys. Rev. B 72, 014547 (2005).

[20] Our experiment does not preselect for realizations that did not tunnel prior to tomography. Thus the probabilities $P_{X}$, $P_{Y}, P_{Z}$ include a background $P_{B}$ population. This population does not respond to the tomographic rotations, and can therefore be subtracted.

[21] M. Riebe et al., New J. Phys. 9, 211 (2007).

[22] Matthew Neeley et al., Nature Phys. 4, 523 (2008).

[23] Radoslaw C. Bialczak et al., Abstract: P33.00010, APS March Meeting (2007).

[24] We use the experimentally measured initial states as input states for the process tomography (and do not assume the ideal states).

[25] For $p>0.9$ the error $p_{r}$ dominates the analysis, causing the fidelity shown in Fig. 4(b) to become unphysical. 